\title{
Predicting personal thermal preferences based on data-driven methods
}

José Joaquín Aguilera ${ }^{1, *}$, Jørn Toftum ${ }^{1}$, and Ongun Berk Kazanci ${ }^{1}$

${ }^{1}$ International Centre for Indoor Environment and Energy, Technical University of Denmark, 2800 Kgs. Lyngby, Denmark

\begin{abstract}
One of the prevalent models to account for thermal comfort in HVAC design is the Predicted Mean Vote (PMV). However, the model is based on parameters difficult to estimate in real applications and it focuses on mean votes of large groups of people. Personal Comfort Models (PCM) is a data-driven approach to model thermal comfort at an individual level. It takes advantage of concepts such as machine learning and Internet of Things (IoT), combining feedback from occupants and local thermal environment measurements. The framework presented in this paper evaluates the performance of PCM and PMV regarding the prediction of personal thermal preferences. Air temperature and relative humidity measurements were combined with thermal preference votes obtained from a field study. This data was used to train three machine learning methods focused on PCM: Artificial Neural Network (ANN), Naive-Bayes (NB) and Fuzzy Logic (FL); comparing them with a PMV-based algorithm. The results showed that all methods had a better overall performance than guessing randomly the thermal preferences votes. In addition, there was not a difference between the performance of the PCM and PMV-based algorithms. Finally, the PMV-based method predicted well thermal preferences of individuals, having a $70 \%$ probability of correct guessing.
\end{abstract}

\section{Introduction}

The prevalent approach for design of thermal comfort in HVAC systems worldwide is based on the Predicted Mean Vote (PMV) model $[1,2]$. This model predicts the overall thermal sensation of occupants, based on two personal parameters: metabolic rate, clothing level; and four environmental variables: relative humidity, mean radiant temperature, air temperature and air velocity. However, the method requires data that is difficult to estimate in real applications, such as: metabolic activity rate and clothing level. In addition, the PMV is not able to re-learn from new data since the input parameters it uses are fixed in the model. Lastly, the model had a poor predictability performance when applied to individuals in some field studies [3-5]. In the last years, a new approach to model thermal comfort has been suggested, taking advantage of modern data modelling techniques, named Personal Comfort Models (PCM). They take individuals as units of analysis, where measured data is combined with feedback from occupants to create models that predict individual responses [6]. PCM are based on data that is easy-to-obtain and cost-effective, using machine learning algorithms for data processing. Different algorithms and sources of information can be used, adding flexibility to the data modelling.

The framework described in this report evaluates the performance of three different machine learning techniques and compares them with an algorithm grounded on the PMV model. Data obtained from a participatory sensing assessment in two university offices was used to compare all the methods in terms of the prediction of thermal preference votes. This project contributes with the following: (1) A field evaluation of a thermal comfort participatory sensing approach, (2) A performance evaluation of four methods: Artificial Neural Networks (ANN), Naive-Bayes (NB), Fuzzy Logic (FL) and Predicted Mean Vote (PMV) with regards to thermal preference predictability.

\section{Related work}

Different approaches to model thermal comfort at a personal level have been made in recent years. Many of the initial attempts originated from multidisciplinary efforts rather than thermal comfort research alone. A number of those studies used the PMV index as the metric to integrate thermal comfort in learning algorithms [7-10]. All of them employed a multi-valued logic called fuzzy logic to characterize different thermal comfort categories given by the PMV. This approach has the limitations of the PMV model: the difficulty to account for personal parameters and is not focused on individuals. As a result, there is a growing interest to develop methods that employ data easy and cheap to measure, taking advantage of stateof-the-art mathematical modelling methods. Different machine learning techniques have been tried depending on the data availability and the focus of the method. Bayesian networks was the tool implemented by [11] to model thermal comfort preferences. This framework achieved a $70 \%$ accuracy when predicting thermal preference votes from occupants in a field study. The same learning technique was used by [3] to determine comfort temperatures with the ASHRAE RP-884 data base, a set of data used to develop the Adaptive Thermal Comfort Model [12]. The approach showed an improved performance compared to conventional thermal comfort models such as PMV and the Adaptive model. Artificial Neural Networks were implemented by [13] to model thermal sensation. This approach showed $80 \%$ accuracy when predicting occupants' votes in a field evaluation.

Despite the above, there has not been many applications of PCM in field studies for long periods. Fuzzy logic controllers were employed by $[14,15]$ to

\footnotetext{
* Corresponding author: jojap@byg.dtu.dk
} 
model thermal preferences from occupants in offices. That information was used together with ventilation airflow measurements to control a HVAC system for a period of 13 and 14 weeks. The results showed 12-39\% airflow reduction and an improvement of thermal comfort when using the methods based on fuzzy logic. However, the performance of a participatory sensing methodology relies substantially on the degree of participation of the occupants. Keeping the consistency of occupants' participation is a challenging task. Different types of survey interfaces were tested by [16], proposing a plain slider scale that improves participation and consistency when carrying out a participatory sensing approach.

To avoid relying on occupants' feedback, several investigations were made to find correlations between human behaviour and thermal comfort. A Personal Comfort System (PCS) was applied by [6], consisting of a device that allowed occupants to regulate the temperature in their local working area, using a custombuilt seat. Occupants' behaviour when regulating their local thermal environment was combined with surveyed information and thermal environment measurements. This information was used as input to six different PCMbased machine learning algorithms to predict thermal preference votes. The results showed that the PCM had an average prediction accuracy of $73 \%$, which was better than the performance of conventional thermal comfort models, which only yielded $53 \%$ accuracy.

The implementation of PCM in real HVAC applications is still a developing task. More efforts on its standardization and use in practice are needed. How to obtain feedback from occupants on a continuous basis and how to integrate trustful learning algorithms in HVAC control loops are just a couple of the challenges that the research efforts are facing.

\section{Methodology}

A field assessment based on a participatory sensing approach was carried out in two offices at the Technical University of Denmark. Thermal preference votes from six participants were obtained continuously during a period of thirteen days. Occupants were provided with a web-based survey that could be accessed either by smartphones or personal computers. During that period, the thermal environment in the room was modified in a nonsystematic manner by opening windows, turning on/off electric heaters and controlling water flows inside radiators. Air temperature $T a$ and relative humidity $R H$ were recorded periodically every 5 minutes at the local workplace of each occupant by using HOBO-loggers as measuring instruments [17]. This procedure was used to obtain a wide range of thermal preference votes as a result of having different levels of thermal environment inside the offices.

The aim of the evaluation was to characterize the performance of four algorithms when predicting thermal preference categories or classes, generated from the participatory sensing votes. The numerical value of a vote is called Thermal Preference Value (TPV), which can take values between 0 and 18 . Three different classes were generated from the $T P V$ as follows: from 0 to 7 corresponded to "Colder", from 8 to 10 were considered as "No change" and 11 to 18 were considered as "Warmer". A thermal preference category with its corresponding $\mathrm{Ta}$ and $\mathrm{RH}$ measurement formed a data point. The total number of data points gathered along the evaluation period was divided into data used for training and testing the learning algorithms. How good the performance of an algorithm was depended on how well it predicted thermal preference classes based on unseen $\mathrm{Ta}$ and $\mathrm{RH}$ measurements or testing data. An algorithm that has a good performance of predicting thermal preferences is able to provide an accurate description of occupants' individual comfort zones. Thus, HVAC control systems can benefit from the inclusion of such algorithms to provide an adequate indoor environment, specific for different requirements and working conditions.

\subsection{Participatory sensing}

Occupants were asked to answer a simple question: How would you prefer the temperature? The answer was given in a snapping scale, where it was possible to select: much colder, no change, much warmer or any value in between, as shown in Fig.1 (left). After each vote was made, a graphical feedback was given to every participant, illustrated in Fig.1 (right). This plot showed the total number of daily votes per category in the room to encourage occupants' continued participation. All six participants were requested to vote as many times as they could. They were provided with daily reminders during the evaluation period. The only restriction for the participants was not to vote with a minimum time-span of 15 minutes between votes. This condition was to avoid having persistent occupants expecting to get a rapid change of their current thermal environment. However, all votes were taken into account in the assessment, no matter the period of time between them. The design of the participatory sensing survey aimed to maintain participation along the evaluation period and improve consistency, according to the findings of [16].

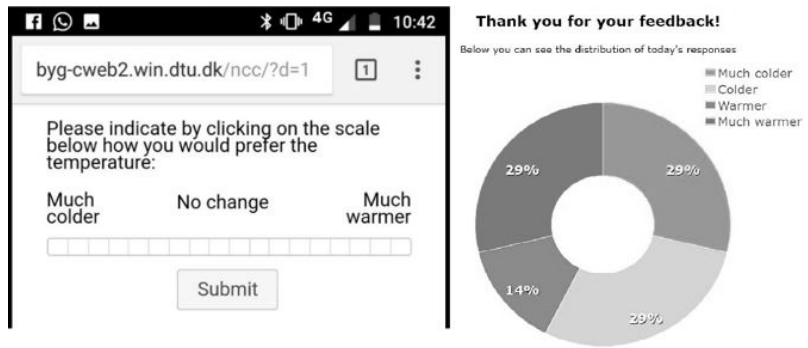

Fig. 1. Survey implemented in the field experiment.

\subsection{Algorithms}

There is a large number of machine learning algorithms available to be applied within PCM frameworks. In this particular article, the chosen methods provided a rather intuitive application and did not consider a large number of assumptions with respect to the data used to train them. 
This allowed implementing the algorithms without adjusting many parameters, thus, it was straightforward to determine their optimal performance. A brief description of the methods and considerations taken into account are presented as follows:

\subsubsection{Artificial Neural Networks (ANN)}

ANN is a method used to solve non-linear problems by using a network composed of individual elements or socalled neurons. In each neuron linear, logarithmic, sinusoidal or other types of mathematical transformations or transfer functions are used. The final result using this technique is a network where the weight of each neuron has been optimized to minimize the error between the output of the network and the data used for training [18]. ANN was implemented by using the Matlab Artificial Network Toolbox and was composed of three types of layers: input, hidden and output. Three different types of transfer functions were tested: Log-Sigmoid (logsig), Hyperbolic-Tangent Sigmoid (tansig) and Linear transformation (purelin). An iterative process was carried out through a method called Levenberg-Marquardt backpropagation (LM-BP). This method adjusts the weights of each neuron to diminish the error between the ANN predictions and the testing data. The process finished either when a maximum of 100 iterations or when a Mean Square Error (MSE) of $10^{-7}$ was reached.

\subsubsection{Naive-Bayes (NB)}

The NB method uses the basic principles of probability, based on the application of Bayes theorem. This states that the probability of a given event is calculated from previous knowledge about conditions related to an event. In particular, the term "naive" comes from the assumption that different factors that affect the event are independent of each other, also named conditional independence. In this method, it is also assumed that all thermal preference categories or classes have the same distribution. To implement this method, first a Probability Density Function (PDF) was selected and applied to the training data, calculating the mean and standard deviation of each parameter. These two statistical parameters were used to calculate the probability of a certain class of unseen data, used for testing [18]. The Matlab Machine Learning Toolbox was applied to develop the NB algorithm.

In both ANN and NB algorithms the overall training process was as follows: (1) The entire data set was read by the algorithm, corresponding to $R H$ and $T a$ measurements and participants' thermal preferences; (2) The data set was standardized based on its mean and standard deviation to eliminate the influence of different orders of magnitude; (3) The data was randomly ordered to eliminate the influence of its arrangement; (4) The entire data set was divided between training and testing data; (5) In NB the standard deviation and mean were calculated as part of the training process, whereas for ANN the weights of all neurons were calculated; (6) The outputs of the algorithms were obtained and compared with the training data.

\subsubsection{Fuzzy logic ( $F L)$}

FL is a multi-valued logic grounded on the statement that the truth of an affirmation is a matter of degree, first introduced by [19]. Unlike in classical logic where a variable can be either 1 or 0 , in FL a variable can also be any value in between those numbers. The data in FL is classified as fuzzy sets, which represent linguistic variables (e.g., hot, cold, low or high). How much a data point belongs to a fuzzy set is given by a membership degree. Unlike in NB and ANN, the FL was only provided with $T a$ measurements. $R H$ was not included since the framework applied to develop the FL algorithm was based on the contribution from [14], who developed an approach grounded on the Wang-Mendel method to create fuzzy logic descriptive models [20]. The FL algorithm in this assessment implemented in Matlab. Three fuzzy sets were assumed, representing the three thermal preference classes: "Warmer", "Colder" and "No change". A Ta value from the training data was considered within a fuzzy set depending on how much its corresponding $T P V$ belonged to that thermal preference category. The three fuzzy sets considered $T P V$ values from 0 to 7 to be "Colder", 8 to 10 for "No change" and 11 to 18 for "Warmer". This allowed having a descriptive model of thermal preferences classes based on measured $T a$. When testing the algorithm, unseen $T a$ values were classified into the different categories depending on their membership degrees. Only the ratio between training and testing data was varied in a sensitivity analysis, evaluating the outcome in terms of classification performance.

\subsubsection{Predicted Mean Vote (PMV)}

The PMV-based method considered that a PMV index below -0.5 corresponds to a preference towards "Warmer", above 0.5 is associated with a preference to the class "Colder" and values between -0.5 and 0.5 indicate a preference of "No change". The implementation of the PMV model was performed by applying in Matlab the algorithm defined in ASHRAE 55 [21]. Three input parameters to determine the PMV index were varied in the method to establish the best performing configuration in terms of classification performance. The clothing level was varied between 0.5-1.2 [clo] accounting for typical garments for summer and winter respectively; the metabolic activity rate between 1-2.1 [met] was tested, corresponding to a range of physical activities that can be performed in offices, from being seated, relaxed to walking; and the mean air velocity was varied between 0 $0.12[\mathrm{~m} / \mathrm{s}]$ representing the maximum range allowed in landscaped offices, according to ISO 7730 [22].

\subsection{Performance evaluation}

Identification of the category or class a new data point belongs corresponds to a classification problem. The algorithms tested in this assessment were evaluated by their capacity to classify thermal preference categories based on thermal environment measurements. How good 
a classification algorithm (or classifier) performed depended on the number of correct and incorrect guesses. When a data point was correctly allocated in a certain category "A", it was called true positive. Similarly, the data that was correctly not allocated in that category was called true negative. On the other hand, the data that was incorrectly classified as "A" was called false positive. Finally, false negatives were data that was supposed to be "A" but was classified in another category. The True Positive Rate (TPR), also named hit rate or recall, is defined as the ratio between the number of true positives and the total number of positives. The False Positive Rate (FPR) or false alarm rate, corresponds to the ratio between the number of false positives and the total number of negatives. TPR states the proportion of positives correctly classified, whereas the FPR gives the probability of wrongly allocating a category as negative. From the relation between both rates it was possible to characterize graphically the performance of a classifier by using the Receiver Operating Characteristics (ROC) [23]. The ROC is a two-dimensional plot, where FPR is placed on the $\mathrm{x}$ axis and the TPR on the y-axis, as shown in Fig.2. This graph represents the trade-off between benefits (true positives) and costs (false positives). A well performing classifier generates larger TPR than FPR, contrary to what happens with a bad classifier. When both ratios are equal, it represents the strategy of randomly guessing a class (dashed line in Fig.2).

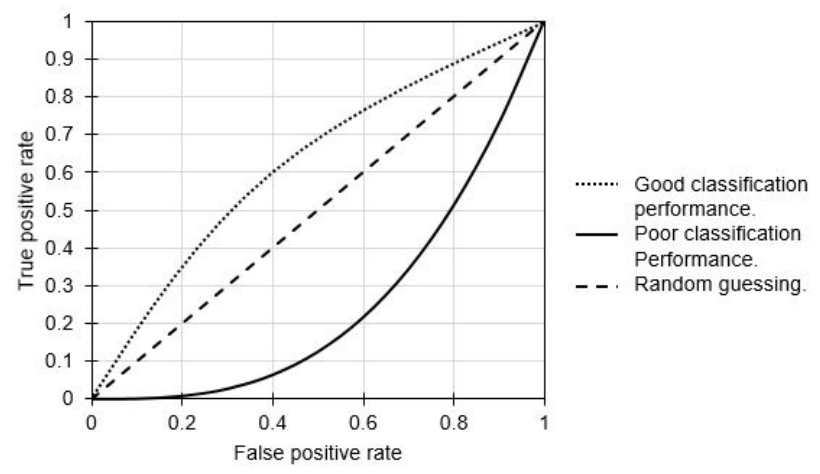

Fig. 2. ROC curve example.

The analysis of the classification performance in the framework presented in this report is based on the Area Under the Curve $(A U C)$, which is a scalar number that simply represents the area under the ROC curve. The $A U C$ is equivalent to the probability that a classifier will rank a randomly selected positive event higher than a negative selected one, i.e., the probability that a class will be correctly classified as such [23]. It can take values between 0 and 1 , corresponding to the minimum and maximum a classifier can perform. For a random guessing classifier the $A U C$ will be 0.5 . Accordingly, values above 0.5 are generated by well performing classifiers and below 0.5 for poorly performing ones. As the aim of the algorithms evaluated in this report was to guess three different thermal preference categories, a multi-class $A U C$ was taken into account. This approach calculates the average $A U C$ of all classes, considering a method called "each class against the rest", represented in Eq. 1 [24].
This method assumes that all classes have uniform distribution, calculating the probability of classifying correctly a class against the others, which is then averaged with the probability from the rest of the classes.

$$
A U C_{m c}=\frac{\sum_{j=1}^{c} A U C\left(j, \text { rest }_{j}\right)}{c}
$$

Where $A U C_{m c}$ is the multi-class area under the curve, $c$ is the total number of classes, $j$ is a class and rest $t_{j}$ represents all the classes different from class $j$.

\section{Results and discussion}

During the survey period, occupants were not forced to participate nor to provide a specific number of votes to avoid influencing their everyday activities. Thereupon, the number of votes per participant along the surveyed period varied considerably (Fig. 3). In spite of the daily reminders and the simplicity of the survey, a decreasing trend in the number of daily votes provided was observed.

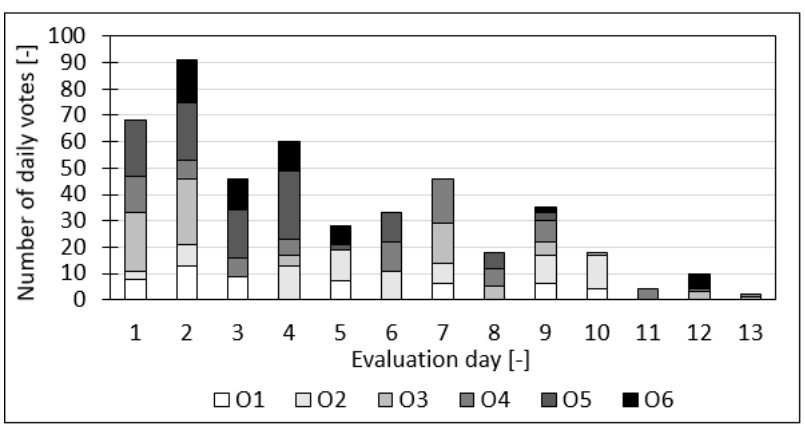

Fig. 3. Number of daily thermal preference votes provided by each occupant along the evaluation period.

Table 1 illustrates the statistical characteristics of the $T P V$ resulting from the assessment. The table shows a lack of variability in the votes, considering that occupants could vote within the $T P V$ range between 0 and 18 . A narrower range of $T P V$ was obtained because of the reduced variation in the air temperatures (Tables 1 and 2). The percentiles show that the votes were mainly biased towards low TPV associated to the category "Colder". This result suggests that the occupants were in general more affected by warmer temperatures in the room than the opposite. Thus, the data provided to the algorithms was not equally distributed among the three classes considered, a problem called imbalanced data. In addition, the percentiles reflect that the classes were not uniformly distributed, i.e., the probability of predicting a vote within a class was not constant. As described by [24], uniform distribution is a basic assumption to evaluate the classification performance of an algorithm by using the multi-class $A U C$ described in Eq.1. In practice, it is difficult to have approximately the same number of TPV values in each class per occupant. Occupants would need to be exposed to different thermal environment conditions during equal periods of time when obtaining the training data. It is therefore a challenging task to characterize accurately the classification performance of a learning algorithm that aims to predict occupants' thermal preferences. 
Table 1. Statistical parameters of the $T P V$ per occupant obtained in the participatory sensing assessment. O: Occupant, STD: Standard deviation.

\begin{tabular}{llllll}
\hline O & $\begin{array}{l}\text { Percentiles } \\
\text { 5/10/90/95 }\end{array}$ & STD & Mean & Median & $\begin{array}{l}\text { Number } \\
\text { of votes }\end{array}$ \\
\hline 1 & $3.0 / 4.4 / 11.0 / 11.0$ & 2.5 & 7.8 & 8.0 & 55 \\
2 & $8.0 / 9.0 / 12.0 / 12.0$ & 1.9 & 9.7 & 9.0 & 80 \\
3 & $5.0 / 7.0 / 10.0 / 10.0$ & 1.7 & 8.6 & 9.0 & 82 \\
4 & $7.2 / 9.0 / 12.0 / 13.0$ & 2.0 & 9.8 & 9.0 & 84 \\
5 & $4.0 / 5.0 / 13.0 / 14.0$ & 3.1 & 9.4 & 9.0 & 110 \\
6 & $4.7 / 6.0 / 14.0 / 14.3$ & 3.1 & 10.3 & 11.0 & 55 \\
\hline
\end{tabular}

The percentiles and standard deviations in Table 1 show that occupants 1,5 and 6 provided votes with higher variability. The feedback from those three occupants were chosen as input data to test the learning algorithms and compare them with the PMV method. The reason was to ensure that all the thermal preference categories had sufficient data points, minimizing the effects of imbalanced data.

Fig. 4 shows the $A U C$ values yielded per algorithm, considering the data of each occupant separately. All methods had a better performance than random guessing $(A U C=0.5)$. Therefore, all classifiers will probably predict more positive instances than negative ones. This shows a good performance considering that only $\mathrm{Ta}$ and $\mathrm{RH}$ measurements were provided to the methods.

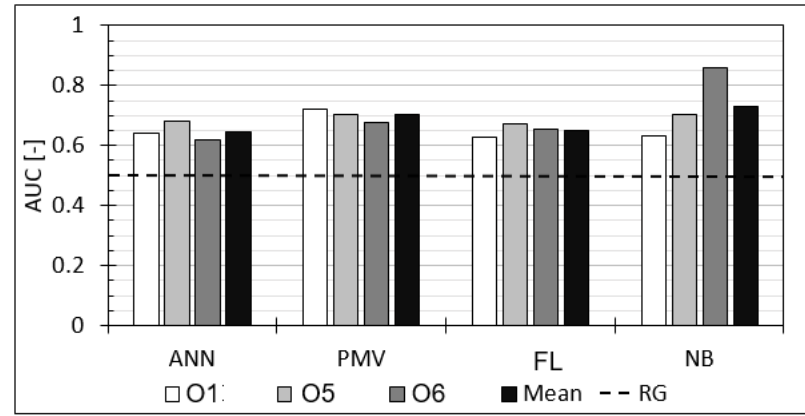

Fig. 4. Classification performance represented by the AUC value for all four algorithms studied, taking into account the data obtained from occupants $1(\mathrm{O} 1), 5(\mathrm{O} 5)$ and 6(O6). RG=Random Guessing line.

The classification performance among the occupants was mainly affected by how many votes per occupant were provided, the distribution of the data points among the classes and the consistency of the votes from the occupants. Higher $A U C$ values could be achieved if any of those factors were improved. The inclusion of data from additional parameters, such as radiant temperature and air velocity, could also improve the classification performance of the algorithms tested.

Overall, the methods with the highest performance were NB and PMV, accounting for a probability of correctly predict a class of $73 \%$ and $70 \%$, respectively. The NB method assumed that $T a$ and $R H$ were independent from each other. It calculated the mean and standard deviation of the training data, adjusting a PDF. Hence, it did not calculate individual factors related to each data point. That was the reason why it performed better than the other algorithms. By calculating variables that comprise a whole data set, it simplifies the learning process.

Fig. 5 shows the performance of all methods with regards to each thermal preference category. Classifying incorrectly a category could yield to serious operational problems when applied in reality. Thermal comfort and health could be compromised when a HVAC control system regulates the thermal environment wrongly. For instance, controlling an indoor environment based on a preference towards colder temperatures instead of warmer, could have serious implications in occupants' well being. Fig. 5 shows that all methods except FL had a better performance when predicting the "No change" category than any other class. This is owed to the unbalanced data among the classes, showed in Table 1.

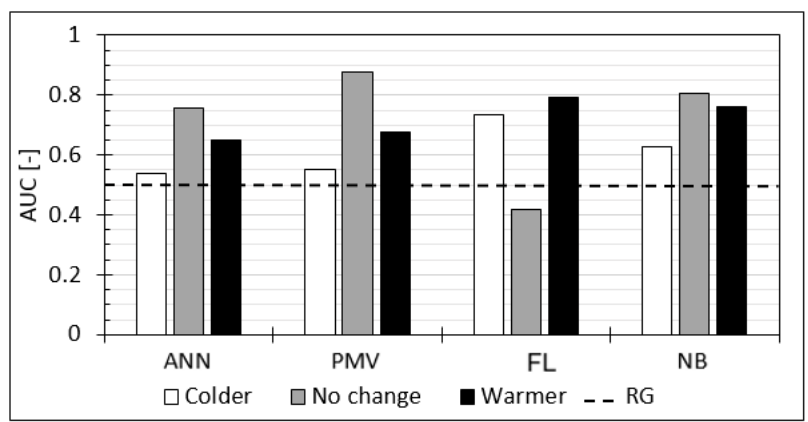

Fig. 5. Classification performance represented by the AUC value for all four classifier studied, taking into account the three thermal preference classes predicted. $\mathrm{RG}=$ Random Guessing line.

Some machine learning methods were more sensitive to imbalanced data than others were. They tended to favor the "no change" class for having the largest proportion of data, translated in a larger amount of true positives. In that context, the NB method exhibited less difference in the prediction of different classes. This method reduced the influence of biased data by assuming that all classes had the same PDF and by calculating parameters that enclose a whole data set. To avoid the problem of imbalanced data, it would be needed to expose people under uncomfortably warm/cold environments for a period equal to the period they feel comfortable. Since the last is unlikely to be applicable in reality, it is desired that the algorithm employed to predict thermal preferences overcomes the problem of not uniformly distributed classes. For that, it is proposed to make a sensitivity analysis of a classifier changing the distribution of the training data per class [25].

A correlation between the amount of training data needed by the learning algorithms and their corresponding classification performance is illustrated in Fig.6. This information allows the identification of how much the number of votes can be decreased with regards to the variation of the performance of a method. The data of all the occupants was combined and a linear correlation was applied for comparison purposes, even though the actual correlation may not be linear. A single data point corresponded to a thermal preference category with its corresponding measurement of $T a$ and $R H$ (only $T a$ for 
Table 2. Parameters that yielded the highest $A U C$ on each algorithm, considering the data from all the three participants. LM-BP: Levenberg-Marquardt backpropagation.

\begin{tabular}{|c|c|c|c|c|}
\hline Algorithm & Parameter & Occupant 1 & Occupant 5 & Occupant 6 \\
\hline \multirow[t]{6}{*}{ ANN } & Ratio training/total data & 0.4 & 0.4 & 0.3 \\
\hline & Number of hidden layers & 3 & 6 & 3 \\
\hline & Training function & LM-BP & LM-BP & LM-BP \\
\hline & $1^{\text {st }}$ transfer function & purelin & purelin & purelin \\
\hline & $2^{\text {nd }}$ transfer function & purelin & purelin & purelin \\
\hline & Number of iterations (epochs) & 3 & 3 & 3 \\
\hline \multirow[t]{2}{*}{ NB } & Ratio training/total data & 0.5 & 0.9 & 0.9 \\
\hline & PDF & normal & normal & normal \\
\hline FL & Ratio training/total data & 0.7 & 0.2 & 0.2 \\
\hline \multirow[t]{3}{*}{ PMV } & Clothing level & 1.1 & 1.1 & 1.2 \\
\hline & Metabolic rate & 1 & 1 & 1 \\
\hline & Air velocity & 0.10 & 0.10 & 0.12 \\
\hline
\end{tabular}

the FL method). Fig.6 illustrates that all the methods had a performance better than random guessing, even when the amount of training data was reduced to only 10 data points. The NB was not only the best performing method, but also required less data to generate a higher AUC compared to the other algorithms. The performance of NB and ANN increased with an increase of the amount of training data, whereas the FL method diminished its performance. Unlike the two other learning methods, FL does not rely on an iterative process to diminish the error during the training process of the algorithm. When training the FL method, the first part of the training data read by the algorithm was used to construct the fuzzy sets. The rest of the training data did not contribute to create better fuzzy sets, as they were already created by the first data points read. Thus, providing more data point to the FL algorithm did not improve its performance.

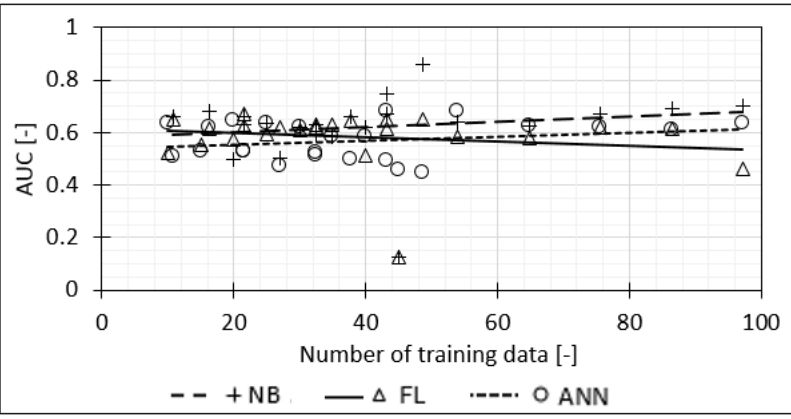

Fig. 6. Classification performance represented by the AUC value as a function of the amount of data required for training on each of the three learning algorithms analysed.

\section{Limitations}

There are a number of limitations with regards to the framework proposed in this assessment. First, the evaluation period considered in the field assessment was limited. A longer period would allow having more input data for the learning algorithms, accounting for variations that the thermal preferences may have with different weather conditions. As a result, the classification performance of the PCM-based algorithms could be analyzed with more training data. Second, miss- classification costs, i.e., the cost of not classifying correctly a category, were not taken into account. In reality, it does not have the same implications to classify a "Warmer" category as "No change" than classifying it as "Colder". This should be taken into account when characterizing the performance of PCM, especially when implemented in real applications. Third, it was considered that $T P V$ was mainly influenced by air temperature and relative humidity. It would be needed to determine the required number of votes per occupant to minimize the influence of other factors that may influence the thermal preference votes. This will help to define the minimum number of votes per occupant needed to ensure a desired classification performance.

\section{Conclusions}

Personal Comfort Models (PCM) allow to focus on the thermal comfort needs of individuals based on local indoor environment measurements and feedback provided by them. Three PCM-based methods were tested in this assessment, based on thermal preference votes obtained from a field study survey. A method based on the PMV model was also calculated and compared with PCM. From the results obtained in this assessment, the conclusions were:

- When predicting personal thermal preferences, all the four algorithms tested (ANN, NB, FL and PMV) showed a better overall performance than guessing randomly, even though only air temperature and relative humidity were provided as input data.

- The difference between the performance of the PCMbased methods and the PMV-based method was very modest.

- The PMV method was capable of predicting thermal comfort at an individual level, with a probability of guessing correctly $70 \%$ of personal thermal preference votes.

- The NB method was not only the best performing method, predicting $73 \%$ of the thermal preferences, but also performed better at predicting each singular thermal preference category, requiring less training data than the other methods. 
The implementation of PCM in field studies is still a developing field. It has the potential to contribute substantially to improve the operation of modern HVAC and BMS systems. Future research efforts will be focused on the implementation of PCM in HVAC control loops, focusing on easy-to-obtain data. A participatory sensing assessment for a longer evaluation period will also be part of the future work in this direction.

\section{References}

1. P.O. Fanger, Mc. Graw Hill (1970)

2. B. Olesen, KC. Parsons, Energy Build., El Sevier, 34, 537 (2002)

3. F. Auffenberg, 2547 (2015)

4. J. Kim, Build. Environ., El Sevier, 132, 114 (2018)

5. J. Van Hoof, Wiley Online Library, 18, 182 (2008)

6. J. Kim, Y. Zhou, S. Schaivon, Build. Environ., El Sevier, 129, 96 (2018)

7. P. Bermejo, L. Redondo, L. de la Ossa, Energy Build., El Sevier, 49, 367 (2012)

8. D. Kolokotsa, G. Saridakis, A. Pouliezos, G. Stavrakakis, Energy Build., El Sevier, 38, 1084 (2006)

9. F. Calvino, M. La Gennusa, G. Rizzo, G. Scaccianoce, Energy Build., El Sevier, 36, 97 (2004)

10. L. Hang, D. Kim, App. Sci., 8, 1031 (2018)

11. A. Ghahramani, F. Jazizadeh, B. Becerik-Gerber, Energy Build., El Sevier, 92, 86 (2014)

12. R. De Dear, G.S. Brager, Transactions, ASHRAE, 104, 145 (1998)

13. W. Liu, Z. Lian, B. Zhao, Energy Build., El Sevier, 39, 1115 (2007)

14. F. Jazizadeh, A. Ghahramani, B. Becerik-Gerber, Energy Build., El Sevier, 70, 398 (2014)

15. A. Ghahramani, F. Jazizadeh, B. Becerik-Gerber, Energy Build., El Sevier, 85, 536 (2014)

16. F. Jazizadeh, F. Marin, B. Becerik-Gerber, Energy Build., El Sevier, 68, 140 (2013)

17. C. C. Onset, https://www.onsetcomp.com (2018)

18. M. Mørup, M.N. Schmidt, M. Mørup, Technical University of Denmark (2018)

19. L. Zadeh, Trans. on fuzzy syst., IEEE, 4, 103 (1996)

20. L. Wang, J. Mendel, Trans. On syst., man and cyb., IEEE, 22, 1414 (1992)

21. A.S.H.R.A.E., Standard 55-2013 (2013)

22. E. ISO, Standard 7730:2005 (2005)

23. T. Fawcett, Pat. Rec. Let., El Sevier, 27, 861 (2006)

24. C. Ferri, J. Hernandez-Orallo, R. Modroiu, Pat. Rec. Let., El Sevier, 30, 27 (2006)

25. H. He, E. Garcia, Tran. On Know. Dat. Eng., IEEE, 21, 1263 (2009) 